\title{
Properties of Infrared Source Based on the Big Data of LAMOST Spectral Survey
}

\author{
Le Tian ${ }^{1,2^{*}}$, ZhongZhong Zhu ${ }^{1,2}$, Liyun Zhang ${ }^{1,2}$ and Shuai Wang ${ }^{1,2}$ \\ ${ }^{1}$ College of Science\&College of big data and information engineering, Guizhou University, Guiyang 550025, P. R. \\ China \\ ${ }^{2}$ College of big data and information engineering, Guizhou University, Guiyang 550025, P. R. China \\ Email: liy_zhang@hotmail.com
}

\begin{abstract}
Big data of the spectroscopic survey of the Large Sky Area Multi-object Fiber Spectroscopic Telescope (LAMOST) are important for studying the properties of infrared source. We obtained 5946 spectra of 4843 infrared stars through cross matching of LAMOST DR3 and WISE. We measured the equivalent widths of the $\mathrm{H} \alpha$ line and other Balmer lines, Ca II H and IRT lines. According to the EWs of $\mathrm{H} \alpha$ lines, we found there are 390 spectra of 294 infrared stars showing strong activity. We found that 77 spectra were first observed by LAMOST. We found 36 objects show chromospheric activity variation in the $\mathrm{H} \alpha$ emission line. In the end, we gave the physical mechanism of the early-type stars and late-type stars activity.
\end{abstract}

Keywords: infrared source, stars, stellar chromospheric activity, LAMOST

\section{Introduction}

In the Universe, the objects radiating in the infrared band are called infrared sources. There are lots of dust, atoms and molecules in the interstellar space. The main reason for interstellar extinction is that these substances will scatter and absorb electromagnetic radiation, and the dust is the most significant. The observation has shown that the selection of the interstellar extinction wavelength is more significant the ultraviolet and visible light than the infrared extinction. On this account, infrared observation objects that are more faint has more advantages.

The star also has infrared radiation in the process of evolution. The typical star-forming region are infrared dark clouds, IRDCs. It is considered as a massive star-forming region (Rathborne et al., 2006; Peretto et al., 2010). IRDCs are cold, highly invisible, dense molecular clouds, which are firstly observed in the bright background disappearance in infrared galaxy (Perault et al., 1996; Egan et al., 1988). When the star evolves to the pre-main sequence and main sequence star stages, its infrared radiation is mainly from the circumstellar dust disk and planet disk. The radiation of the dust received from the central star is in the infrared band. The observation proves that the circumferential disk around the main sequence star is a common phenomenon, IRAS unexpectedly found infrared excess around in Vega (Aumann et al., 1984). Using the observation results of IRAS, Oudmauher (1992) found 462 infrared excess in the SAO star table and verified that the celestial bodies have PMS, Be, AGB and the original planetary systems. Rhee (2007) selected 160 infrared excess from the IRAS observation database to select debris disk distance about $120 \mathrm{pc}$. The spectra of the central celestial bodies are mostly early-type stars of B-F and only a few of them are late type star.

Yet the most interesting thing was stellar magnetic activity and chromospheric activity. The rule of its activities will produce a set of emission lines in the spectrum of stars, representative of the Balmer line, Ca II H\&K, and Ca II infrared triple line. It is generally believed that the magnetic reunion and the deep convection are the reasons for this phenomenon (Zhang et al., 2015; Vida et al., 2015; Zhang et al., 2016; Montes \& Crespo-chacón, 2004). Catalano (1998) revealed the relationship between the $\mathrm{H} \alpha$ and Ca II lines according to solar results. Frasca (2016) calculated the $\mathrm{H} \alpha$ and Ca II IRT fluxes of cool stars $\left(T_{e f f} \leq 6000 \mathrm{~K}\right)$ have found 442 the chromospheric of active stars. Yi et al. (2014) found $1971 \mathrm{H} \alpha$ active star from $58360 \mathrm{M}$ dwarfs in the LAMOST pilot survey. Therefore, it is of great significance to study the spectral properties of the stars in understanding the magnetic activity and chromospheric activity. 
We introduced the LAMOST and WISE survey and data screening in the section 2. Spectral analysis of richly active and poorly active of stars are in section 3. It is also in this part of the spectral type of statistics. In the section 4 , we summarized our results.

\section{Data}

\subsection{LAMOST and WISE Survey}

The Large Sky Area Multi-Object Fiber Spectroscopic (LAMOST, also called the Guo Shou Jing Telescope) with a large aperture and wide field adopts an innovative active optics technique (Cui et al., 2012). It is located in Xinglong observation station of National Astronomical Observatories Of China (NAOC). The unique design of LAMOST enables it to take 4000 spectra in a single exposure at a limiting magnitude as faint as $r=19$ at the resolution $R=1800$, which is equivalent to the design aim of $r=20$ for the resolution $\mathrm{R}=500$ (Zhao et al., 2012). At the beginning of the design, the LAMOST was appointed to the two main tasks: the LAMOST Extra Galactic Survey (LEGAS) and the LAMOST Experiment for Galactic Understanding and Exploration (LEGUE) survey of MilkyWay stellar structure. Its appearance effectively promotes international exchanges and cooperation in the field of astronomy.

The LAMOST pilot survey released 906420 spectra totally, containing 807575 stars. Its formal regular survey launched on September 28, 2012 (Luo et al., 2012). The data set of LAMOST data released three (DR3), there are 1622344 total spectral, containing 1489013 stars. In this paper, we found active stars in the stellar catalog of the DR3.

The wide-field Infrared survey Explorer, known as WISE (Wright et al., 2010), is a highly sensitive, all-day sky survey telescope launched by NASA on Dec. 14, 2009. From January 14, 2010 to July 17, 2010 , it completed its first round of patrol, with a central wavelength of 3.4, 4.6, 12 and 22 microns, with a corresponding resolution of $6.1,6.4,6.5,12$ arcseconds, sensitivity $0.08,0.1,1$ and 6 mJy, the source location accuracy of high SNR is even 0.15 arcseconds. WISE works in the mid-infrared band, making many contributions to find young stars. Koenig (2012) identified 3 open clusters and 11 outer Galaxy massive star-forming regions from WISE's survey data and counted the distribution of these young stars in each region. Koenig (2015) had certified 418 stars in $\sigma$ Orionis and 544 stars in $\lambda$ Orionis from the WISE-2MASS catalogs of YSO candidates.

\subsection{LAMOST and WISE Cross-Certification}

In this section, we will introduce the data selection process. Our purpose is to find active stars through data cross-certification released by the LAOMST DR3 and the WISE all-sky survey. As described above, the LAOMST DR3 have 1489013 stars. And the WISE all-sky survey released 345165 infrared source, which contains 158114 stars, 145905 galaxies and 41146 other types of celestial bodies. The key of crosscertification is that the observation coordinates of the same source of two telescopes are within the error range. Therefore, the error of the rectascension and the declination was set in less than 2 degrees. Through such process, we found 3762 stars in infrared stellar catalog and found 2184 stars in infrared galactic. The LAMOST identified the objects by doing spectrum of $3700 \AA<\lambda<9100 \AA$ (zhao et al., 2012; Cui et al., 2012), and the WISE is photometry in W1 W2 W3 W4 of mid-infrared and far-infrared (Wright et al., 2010). By contrast, the LAMOST identifies more accurately. According to the spectral characteristics exhibited by active stars, that is the $\mathrm{H} \alpha, \mathrm{H} \beta, \mathrm{H} \delta, \mathrm{H} \gamma$ and $\mathrm{Ca}$ II $\mathrm{H} \& \mathrm{~K}$, Ca II IRT, we obtained 390 spectral of active stars from LAMOST's 5946 spectrum. We showed some cross-certification data in Table 1.

\section{Discussion}

\subsection{Active Indicators}

The 390 spectra provided by LAMOST, not all of them, are all characteristic spectral lines of stellar activity. Depending on the nature and extent of the activity of the star, the emission lines on the spectrum vary in degree. Therefore, we chose 8 spectrum of active stars to show, which is signal-to-noise 
Table 1. LAMOST and WISE cross-certification

\begin{tabular}{|c|c|c|c|c|c|}
\hline LAMOST name & WISE name & Spectral S/N & Spectral type & LAMOST Class & WISE Class \\
\hline J000013.42+402929.7 & J000013.43+402929.7 & 710.51 & M1 & star & star \\
\hline J000013.42+402929.7 & J000013.43+402929.7 & 488.93 & M1 & star & star \\
\hline J000013.42+402929.7 & J000013.43+402929.7 & 618.29 & M1 & star & star \\
\hline J000013.92+373735.0 & J000013.93+373735.1 & 677.61 & M1 & star & galaxy \\
\hline J000014.01+373735.2 & J000013.93+373735.1 & 251.97 & M0 & star & galaxy \\
\hline J000016.56+445819.4 & J000016.46+445819.4 & 96.43 & M7 & star & star \\
\hline J000053.81+050500.2 & J000053.81+050500.2 & 3.19 & M0 & star & galaxy \\
\hline J000055.74+175204.4 & J000055.75+175204.3 & 725.96 & bad & star & star \\
\hline J000109.35+425412.2 & J000109.04+425412.0 & 82.4 & M2 & star & star \\
\hline J000112.28+430131.3 & J000111.94+430131.3 & 97.17 & K5 & star & star \\
\hline J000219.16+125818.1 & J000219.14+125818.1 & 45.38 & $\mathrm{~K} 2$ & star & galaxy \\
\hline J000219.16+125818.1 & J000219.14+125818.1 & 65.68 & $\mathrm{~K} 4$ & star & galaxy \\
\hline J000221.87+042937.7 & J000221.65+042937.8 & 258.78 & M2 & star & star \\
\hline J000228.06+414202.5 & J000228.06+414202.6 & 51.46 & $\mathrm{~F} 2$ & star & star \\
\hline J000230.94+382639.3 & J000230.92+382639.3 & 253.48 & M2 & star & star \\
\hline J000253.70+545257.7 & J000253.70+545257.7 & 109.23 & $\mathrm{~K} 4$ & star & galaxy \\
\hline J000307.65+553345.7 & J000307.27+553346.5 & 20.3 & M2 & star & star \\
\hline J000309.51+440930.8 & J000309.52+440930.7 & 50.47 & M6 & star & star \\
\hline J000314.92+160843.0 & J000314.90+160843.2 & 13.99 & M1 & star & galaxy \\
\hline J000333.98+263859.8 & J000334.00+263900.0 & 16.6 & bad & star & galaxy \\
\hline J000336.27+032006.5 & J000336.26+032006.6 & 3.09 & K7 & star & galaxy \\
\hline J000348.79+472927.0 & J000348.79+472927.1 & 689.29 & $\mathrm{~K} 7$ & star & star \\
\hline J000350.15+102703.6 & J000350.22+102703.9 & 480.74 & M2 & star & star \\
\hline J000412.98+104726.0 & J000412.96+104726.1 & 24.61 & K3 & star & galaxy \\
\hline J000412.98+104726.0 & J000412.96+104726.1 & 20.52 & K4 & star & galaxy \\
\hline J000415.54+331821.3 & J000415.52+331821.6 & 6.6 & bad & star & galaxy \\
\hline J000420.07+400635.8 & J000419.99+400634.7 & 576.09 & & star & star \\
\hline J000451.74+120702.2 & J000451.74+120702.3 & 2.35 & M2 & star & star \\
\hline J000548.58+420211.8 & J000548.84+420213.0 & 165.89 & M5 & star & star \\
\hline J000551.15+405739.4 & J000551.15+405739.4 & 468.33 & M1 & star & star \\
\hline J000552.62+550203.7 & J000552.63+550201.5 & 211.58 & M6 & star & star \\
\hline J000601.03+162707.6 & J000601.06+162707.5 & 696.28 & bad & star & star \\
\hline J000601.07+162707.6 & J000601.06+162707.5 & 496.78 & bad & star & star \\
\hline J000610.90+365419.3 & J000610.89+365419.7 & 249.29 & M3 & star & star \\
\hline J000617.12+351113.7 & J000617.12+351113.7 & 3.36 & M0 & star & galaxy \\
\hline
\end{tabular}

Note:Here we show only partial data, and the rest of the data will be uploaded to the web 
ratio and the emisson line is obvious. Figure 3 shows the four infrared source spectra of single observation LAMOST J043157.79+182136.9 LAMOST J051645.45-015122.3 LAMOST J053243.05+122108.4 LAMOST J053913.00-012721.2. Figure 5 shows four infrared source spectra of multiple observations LAMOST J000936.85+374731.9 LAMOST J043029.61+242644.9 LAMOST J040559.62+295638.2 LAMOST J065311.55+113256.3.

\subsection{Stellar Active Statistics}

Active stars range from early stars to late stars. In order to find the law of the active star, we should have been carrying out the statistics of infrared stars. Figure 1 shows that we get the histogram of infrared star statistics. From figure 1, the infrared stars are later than the early stars. Through cross-certification, we obtained 15 B-type stars (4 of them are active), 97 A-type stars (21 of them are active), 251 F-type stars (21 of them are active), 581 G-type stars (27 of them are active), 1361 K-type stars (39 of them are active) and 2667 M-type stars (231 of them are active). In terms of data, whether it's active or not, infrared stars have gradually increased from early stars to late stars, and have the most M-type stars. So this paper mainly discusses the active infrared star optical properties and its chromospheric activity, we do not have an in-depth discussion at one point. It requires more statistical data to prove whether infrared stars have this rule. We shows in figure 2 that the percent of each type of active stars to obtain more in-depth understanding, in which B-type stars are 1.23 percent, A-type stars are 6.46 percent , F-type stars are 6.46 percent, K-type star are 8.31 percent, G-type star are 12 percent, M-type star are 65.54 percent in all the active infrared stars. Why are M-type star so much? Deep convective zones and fast rotation in the chromosphere of many late-type stars produce plages or flares. This phenomenon easily produce balmer lines and Ca II H\&K, Ca II IRT lines to explain active M-type star so much (Zhang et al., 2015; Zhang et al., 2016; Vida et al., 2015; Montes \& Crespo-chacón, 2004).

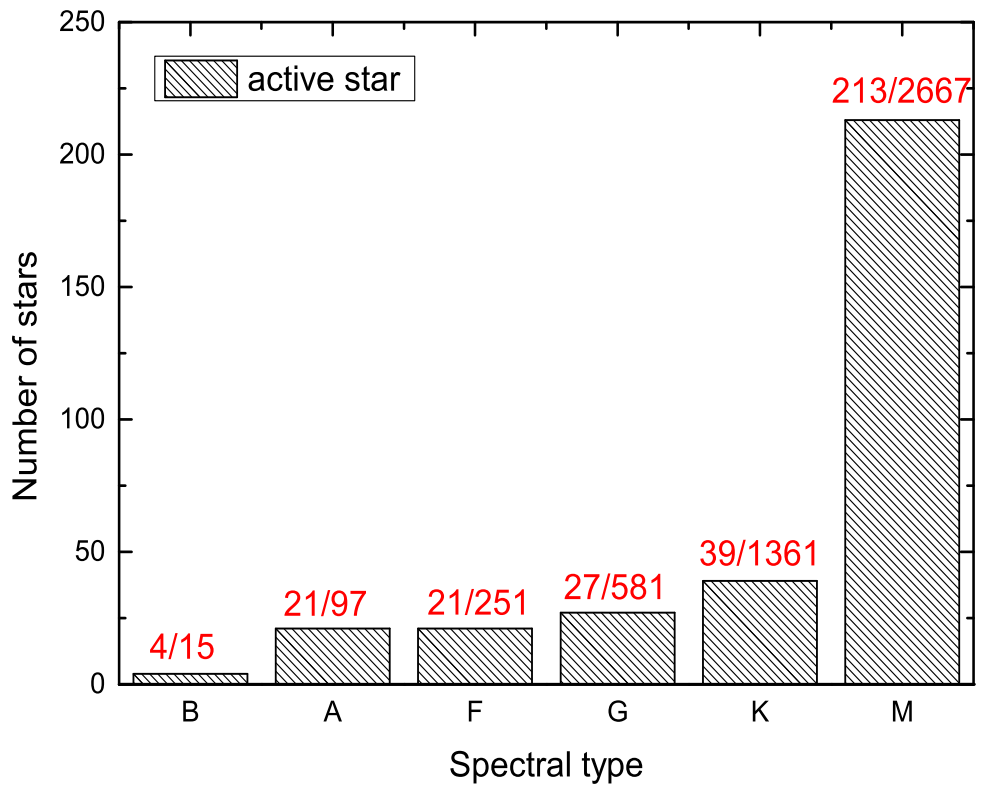

Figure 1. Distribution and proportion of active star with spectral type

\subsection{Active Stars Survey}

The hammer program made by IDL code (Covey et al., 2007) have assigned stellar spectral types, like SDSS. After that, it has been widely adopted by many researchers to match the spectral type of stars 


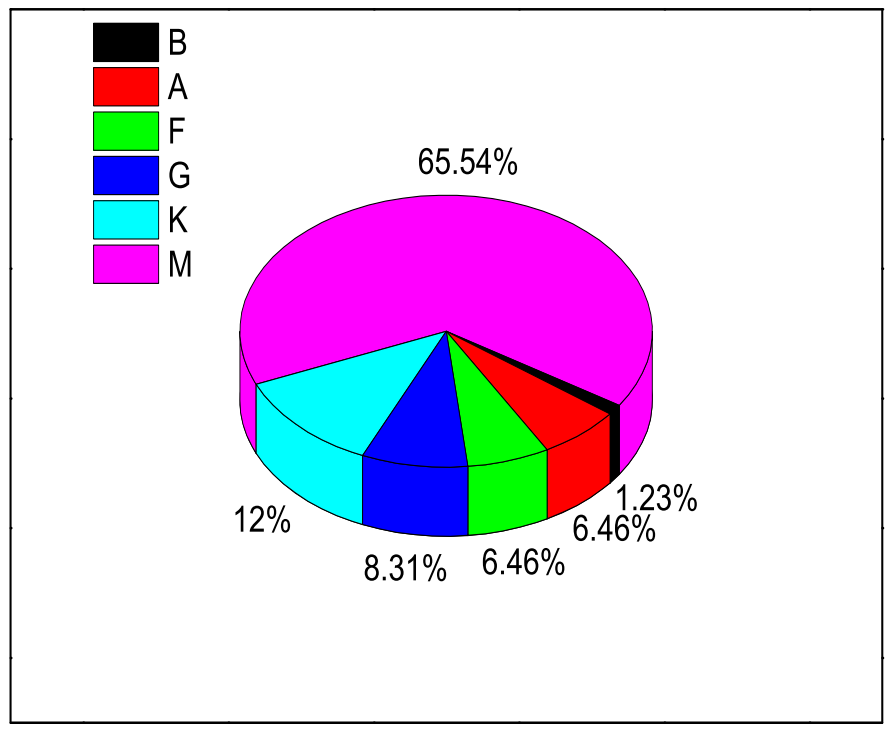

Figure 2. Respectively proportion of each type of active stars

(Zhang et al., 2016; Yi et al., 2014; Lee et al., 2008; West et al., 2011; Woolf \& West, 2012; Dhital et al., 2012). The hammer program is also used. We identified the spectral types of 390 stars and calculated the equivalent width of the $\mathrm{H} \alpha$ line (Hawley et al., 2002; Covey et al., 2007; Zhang et al., 2016). We set the SNR more than 3 in this program. In the hammer program, EWs are calculated by integrating the region of $8 \AA$ wide centered on the $\mathrm{H} \alpha$ line and subtracting away the mean background flux of two

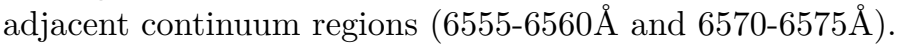

In the Table 2, we shows that infrared source observed with LAMOST. Each of these columns is LAMOST name $(\operatorname{Col}(1))$, date $(\operatorname{Col}(2))$, the $\mathrm{S} / \mathrm{N}$ in LAMOST ugriz bands (from $\operatorname{Col}(3)$ to $\operatorname{Col}(7))$, the magnitudes in WISE W1 W4 bands (from $\operatorname{Col}(8)$ to $\operatorname{Col}(11)$ ), spectral type (Col(12)), research situation $(\mathrm{Col}(13))$, reference $(\mathrm{Col}(14))$, respectively. "-" of the $(\mathrm{Col}(14))$ represented that this infrared source has not been researched in the literature and that is the firstly obtained by the LAMOST. We found that 77 of 390 spectrum firstly observed by looking at the literature. But they have repeated observations of the same source. There are only part of data in Table and the rest of data will be published in electric format. 

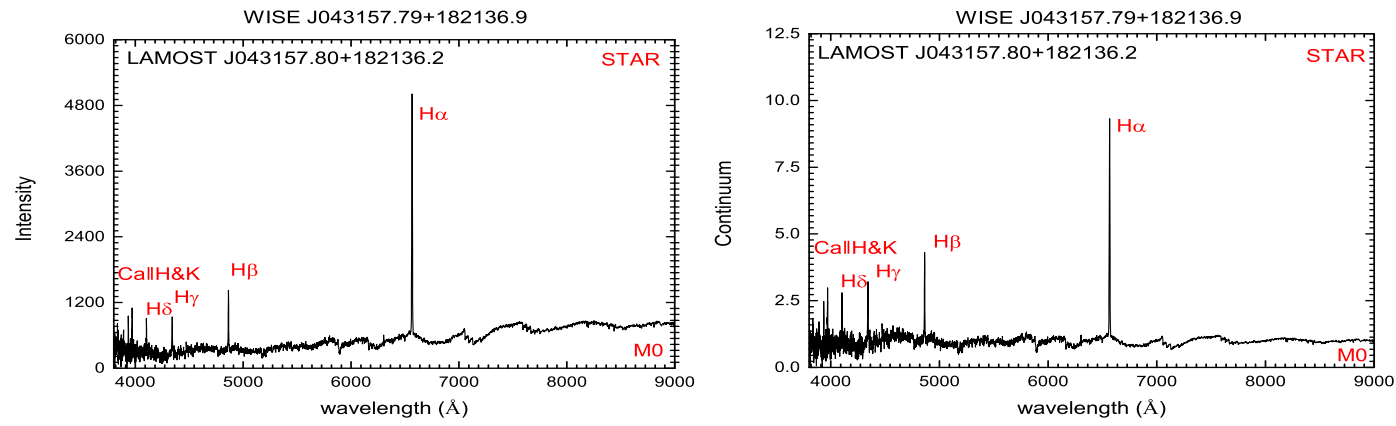

(a) LAMOST J043157.79+182136.9
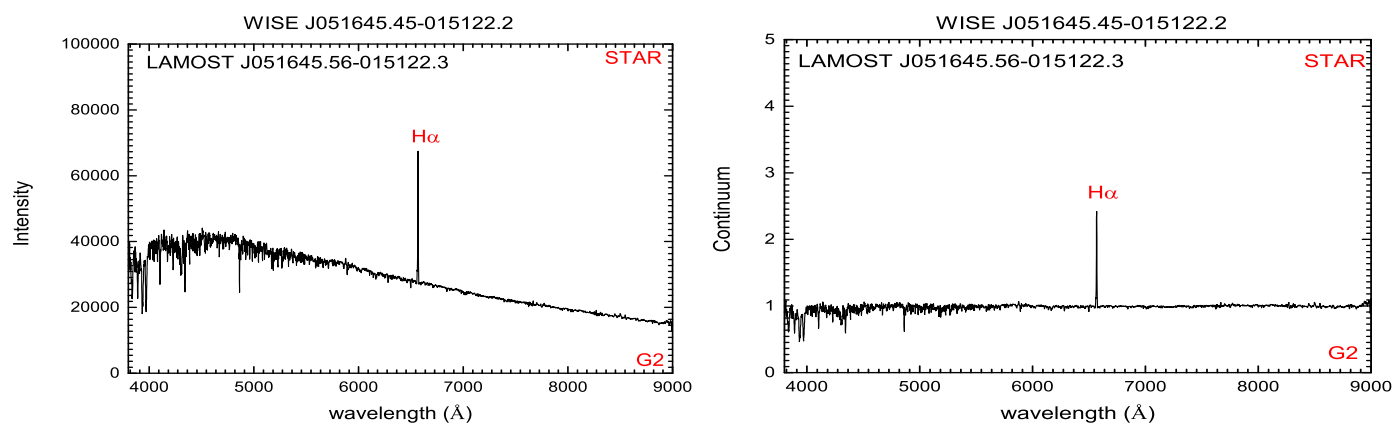

(b) LAMOST J051645.45-015122.3
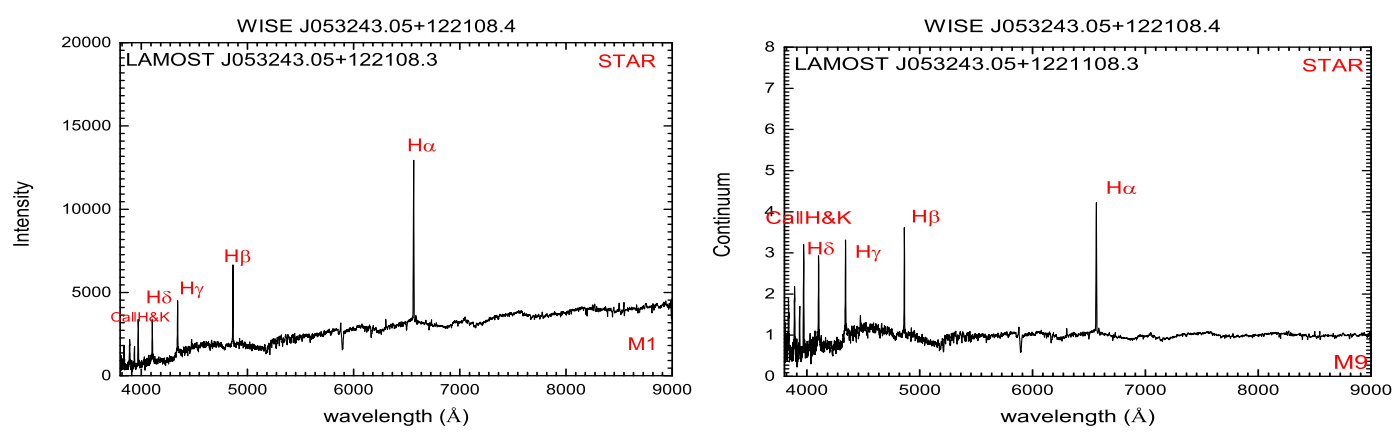

(c) LAMOST J053243.05+122108.4
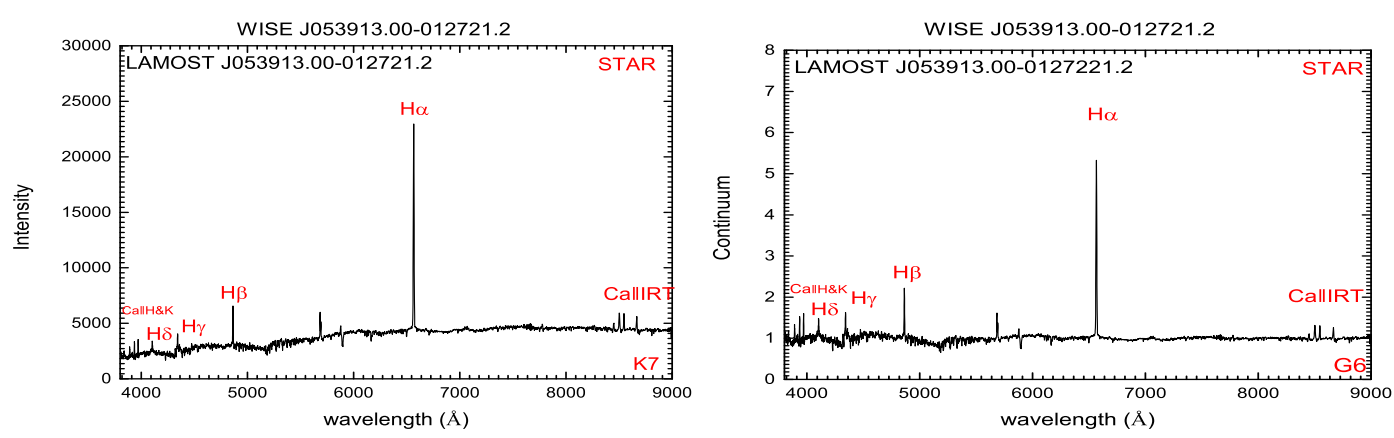

(d) LAMOST J053913.00-012721.2

Figure 3. The spectrum of active infrared stars have $H$ alpha line 

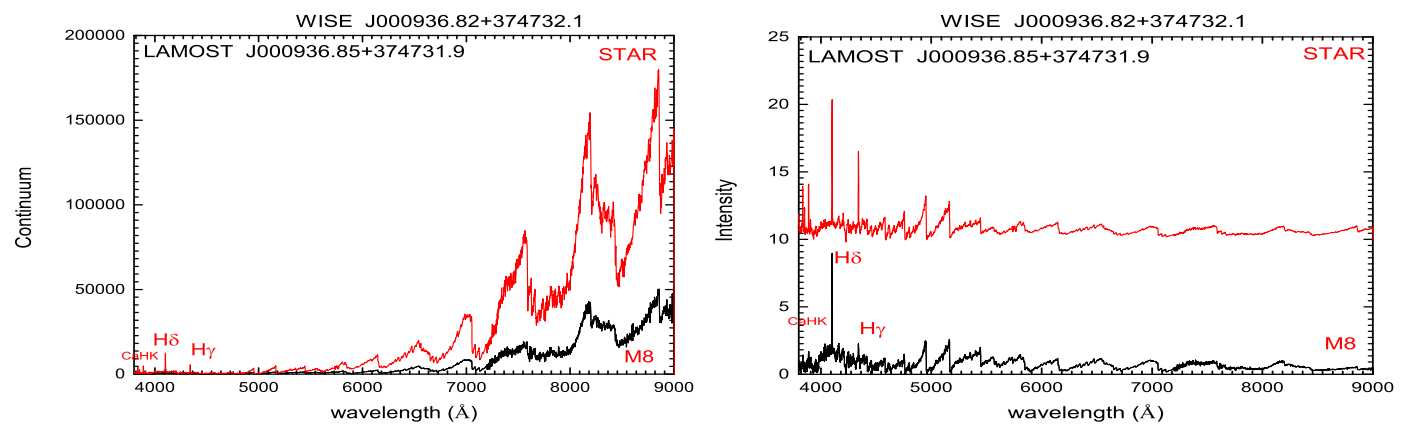

(a) LAMOST J000936.85+374731.9
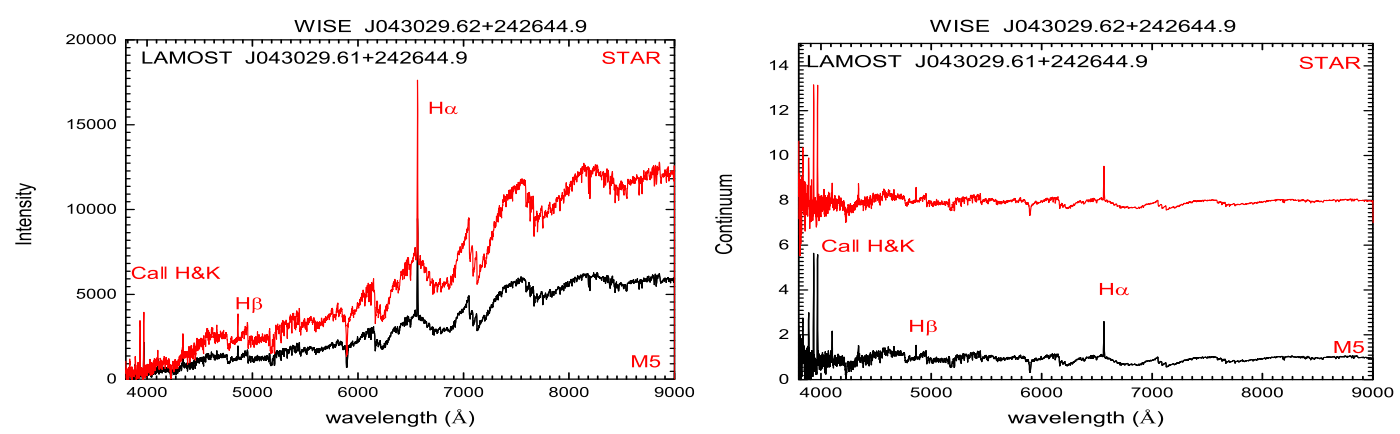

(b) LAMOST J043029.61+242644.9
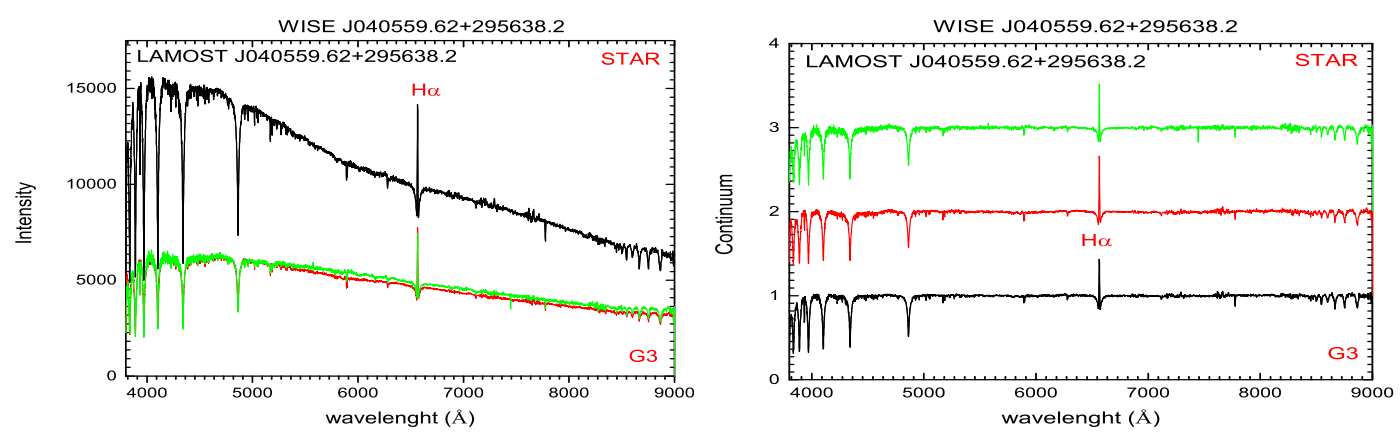

(c) LAMOST J040559.62+295638.2
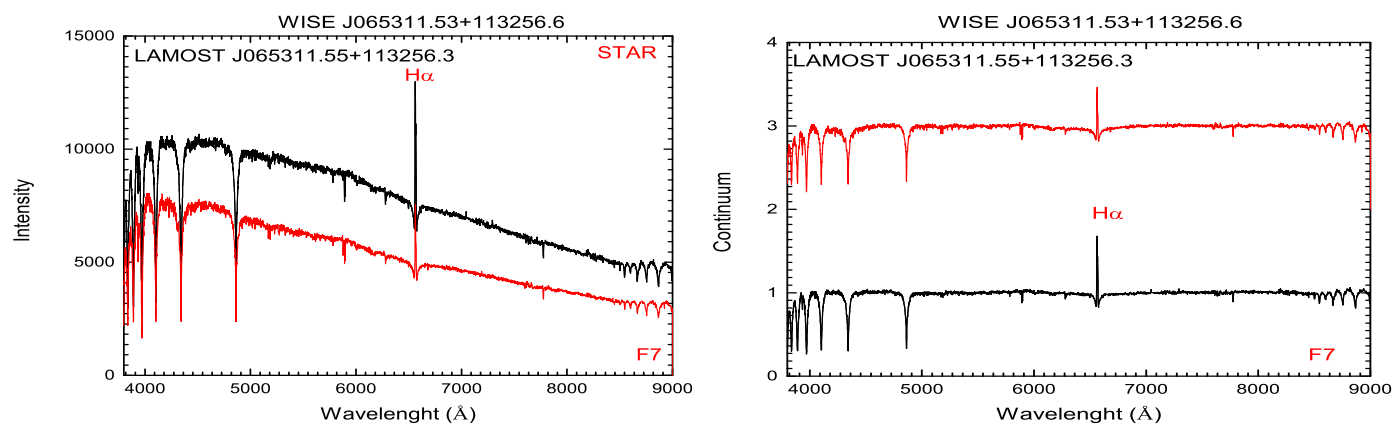

(d) LAMOST J065311.55+113256.3

Figure 4. The spectrum of active infrared stars have twice observations 

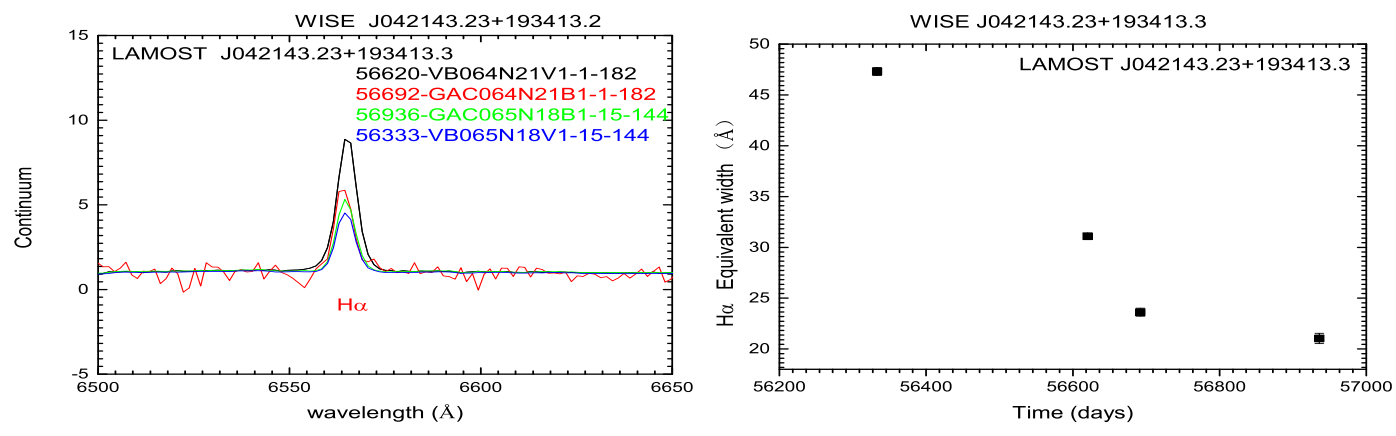

(a) LAMOST J042143.23+193413.3
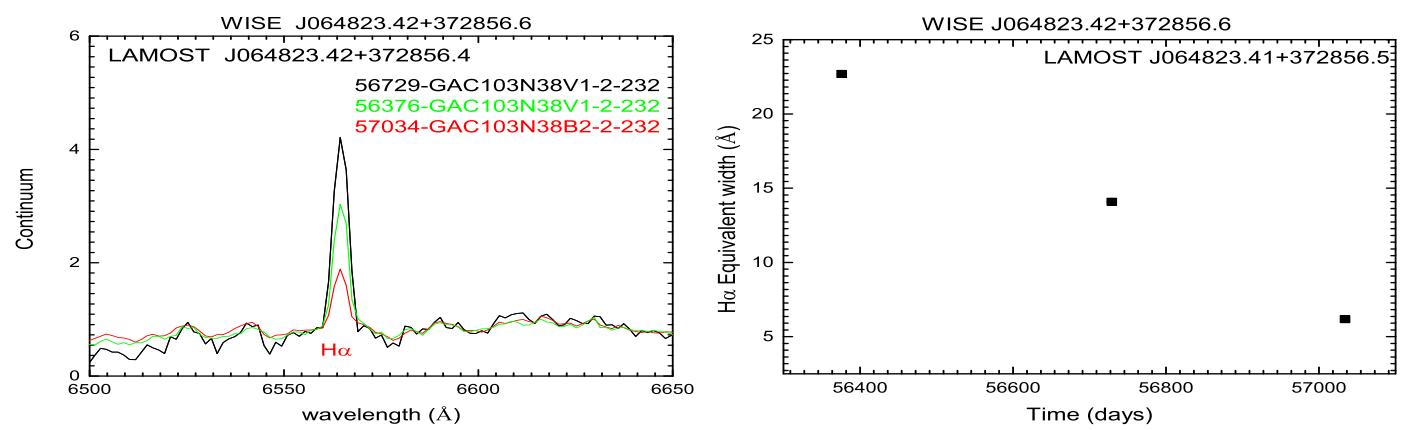

(b) LAMOST J064823.42+372856.6
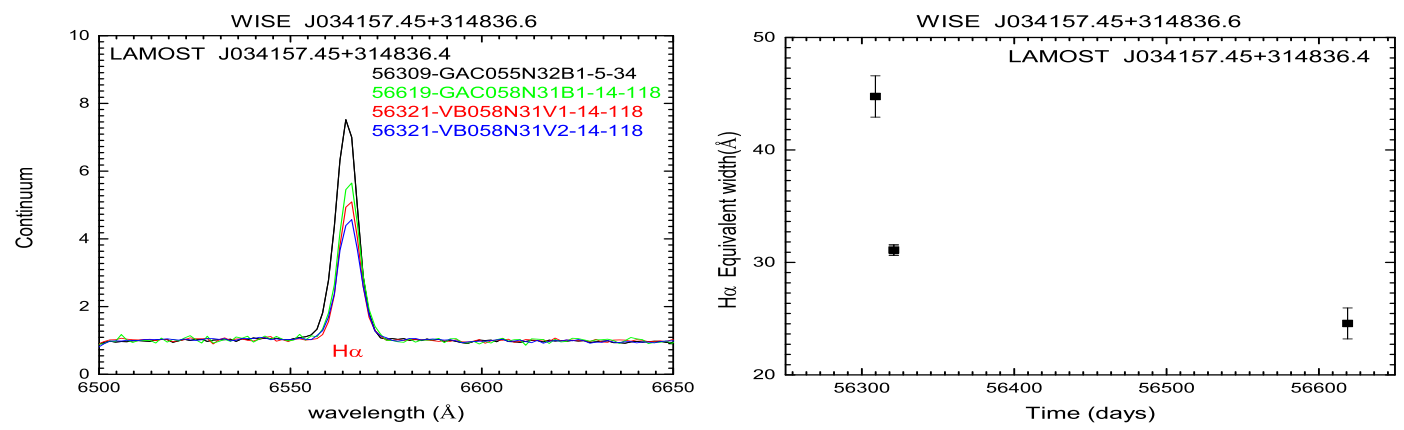

(c) LAMOST J034157.45+314836.4
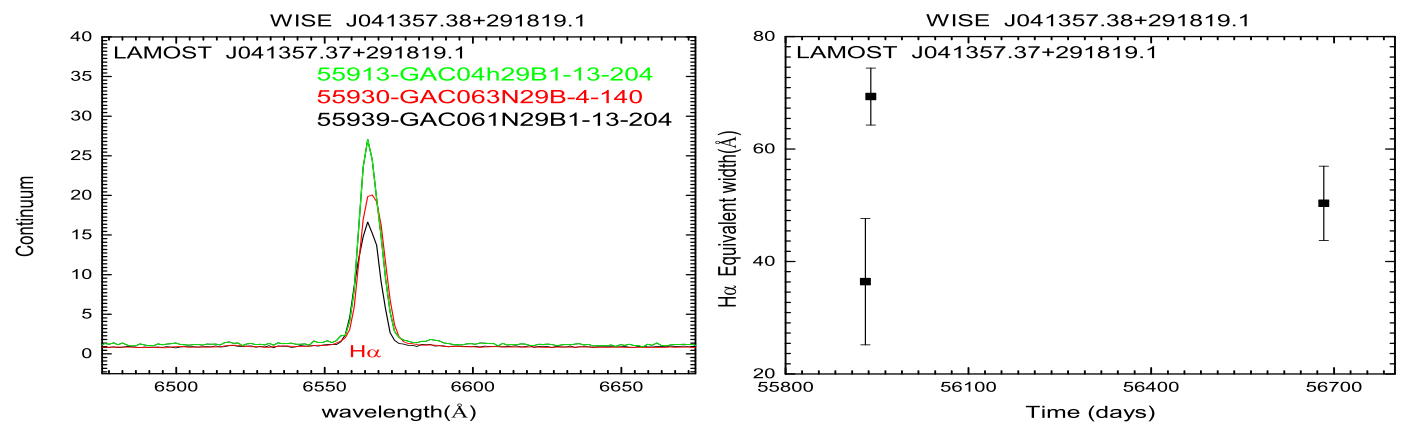

(d) LAMOST J0413573.37+291819.1

Figure 5. Samples of $\mathrm{H}$ alpha The equivalent width is variable. 


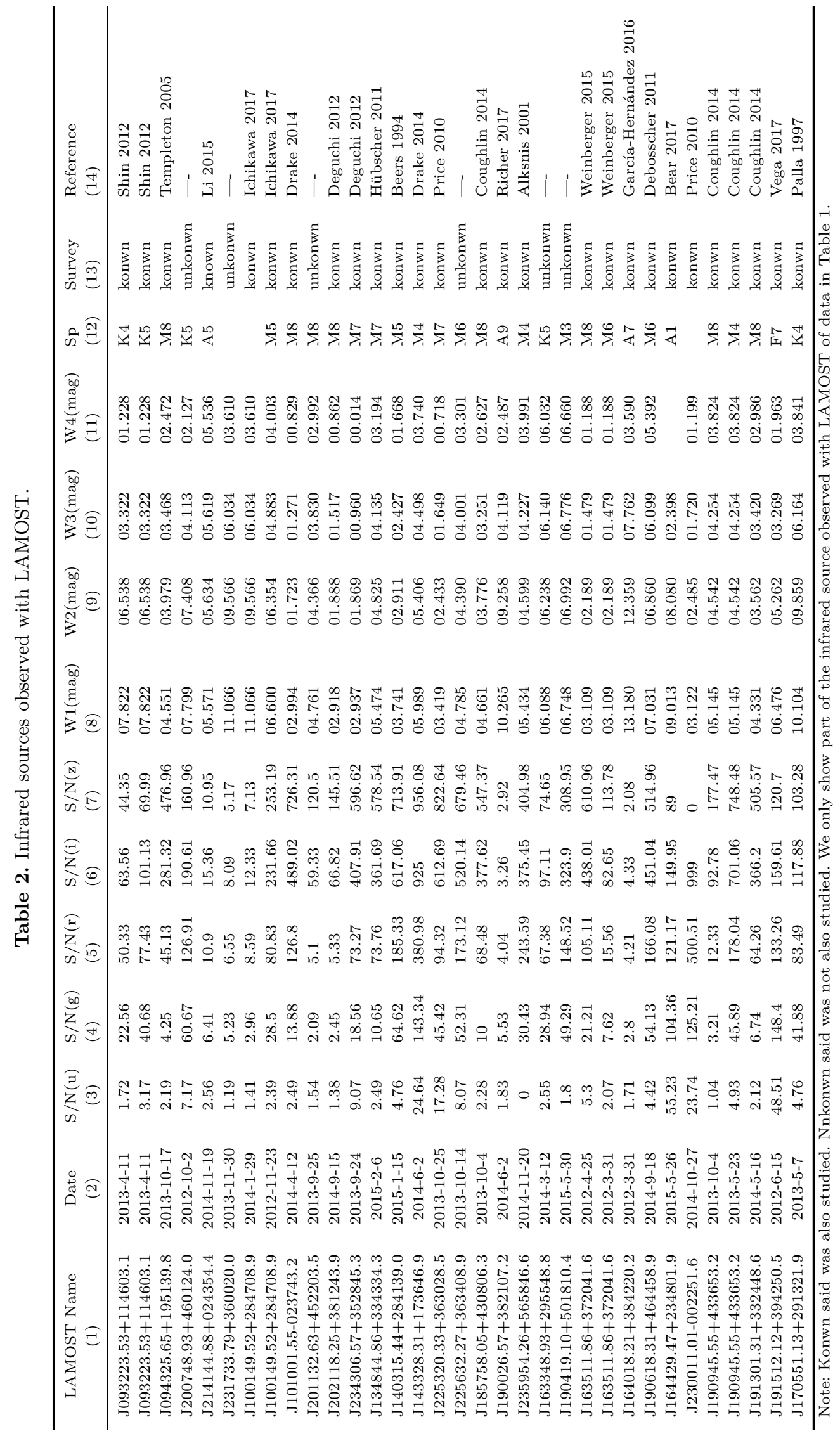


Through hammer program, we found 213 spectrums active stars with the $\mathrm{H} \alpha$ line, in which 116 spectrum have been observed multiple times. To distinction if the changed of multiple observation that chromospheric activity emitted the $\mathrm{H} \alpha$ lines. Our standards are the same as that of West (2011) and Zhang et al. (2016). Firstly the EWs of the $\mathrm{H} \alpha$ lines are no less than $1 \AA$. Secondly the EW of the $\mathrm{H} \alpha$ line should be greater than the value of error. Thirdly the difference of the EW of the $\mathrm{H} \alpha$ line must be three times greater than the error. We found that 102 of them were variable. In Table 3, we shows part of the $\mathrm{EW}$ of the $\mathrm{H} \alpha$ lines.

Table 3. The equivalent width of the $\mathrm{H} \alpha$ line of star with multiple observation.

\begin{tabular}{|c|c|c|c|c|c|c|c|c|}
\hline Same source & LAMOST name & WISE name & Spectral type & $\mathrm{H} \alpha_{e w}$ & $\mathrm{H} \alpha_{\text {error }}$ & Diff & $\mathrm{S} / \mathrm{N}$ & Variable \\
\hline 12 & J041749.65+282935.9 & J041749.66+282936.1 & M2 & 9.8925695 & 0.5173251 & 18.1496849 & 5.2774 & $\mathrm{Y}$ \\
\hline 12 & J041749.65+282936.2 & J041749.66+282936.1 & M5 & 7.1082635 & 0.0690321 & 17.3033504 & 32.9487 & $\mathrm{Y}$ \\
\hline 12 & J041749.65+282936.2 & J041749.66+282936.1 & M5 & 7.1082635 & 0.0690321 & 17.3033504 & 32.7982 & $\mathrm{Y}$ \\
\hline 13 & J041831.12+281628.4 & J041831.13+281628.8 & Mo & 81.6057892 & 1.0935481 & 162.5182953 & 22.1401 & $\mathrm{Y}$ \\
\hline 13 & J041831.12+281628.4 & J041831.13+281628.8 & M2 & 31.5187836 & 5.0577779 & 180.0876770 & 22.0534 & $\mathrm{Y}$ \\
\hline 14 & J041915.83+290626.9 & J041915.83+290626.7 & K4 & 27.0351562 & 2.3639345 & 151.5570831 & 21.5454 & $\mathrm{Y}$ \\
\hline 14 & J041915.83+290626.9 & J041915.83+290626.7 & M1 & 16.6940002 & 0.7525512 & 151.5680237 & 27.3470 & $\mathrm{Y}$ \\
\hline 15 & J042143.23+193413.3 & J042143.23+193413.2 & M5 & 31.0973053 & 0.2316574 & 11.7186966 & 9.1767 & $\mathrm{Y}$ \\
\hline 15 & J042143.24+193413.1 & J042143.23+193413.2 & M4 & 23.6203022 & 0.3863280 & 102.6457443 & 53.0280 & $\mathrm{Y}$ \\
\hline 15 & J042143.24+193413.1 & J042143.23+193413.2 & M3 & 21.0372410 & 0.5113782 & 88.4025040 & 46.2609 & $\mathrm{Y}$ \\
\hline 15 & J042143.23+193413.3 & J042143.23+193413.2 & M5 & 47.2996941 & 0.3964970 & 152.2059021 & 60.3708 & $\mathrm{Y}$ \\
\hline 16 & J042921.64+270125.7 & J042921.65+270125.7 & M2 & 5.5130100 & 0.1473798 & 9.3334055 & 22.3557 & $\mathrm{~N}$ \\
\hline 16 & J042921.65+270125.6 & J042921.65+270125.7 & L1 & 45.0672035 & 14.0986404 & 61.1171722 & 15.6807 & $\mathrm{~N}$ \\
\hline 16 & J042921.64+270125.7 & J042921.65+270125.7 & M8 & 53.1636848 & 14.3353949 & 41.7393112 & 13.0922 & $\mathrm{~N}$ \\
\hline 17 & J042923.73+243300.2 & J042923.73+243300.9 & F4 & 52.8479309 & 2.1284502 & 21.2071342 & 4.2842 & $\mathrm{Y}$ \\
\hline 17 & J042923.73+243300.2 & J042923.73+243300.9 & $\mathrm{K} 2$ & 75.8311081 & 1.7166258 & 20.9036102 & 7.9231 & $\mathrm{Y}$ \\
\hline 18 & J043029.61+242644.9 & J043029.62+242644.9 & $\mathrm{F} 4$ & 52.8479309 & 2.1284502 & 21.2071342 & 47.1404 & $\mathrm{Y}$ \\
\hline 18 & J043029.61+242644.9 & J043029.62+242644.9 & M3 & 9.2656679 & 0.0069753 & 34.4671021 & 46.1454 & $\mathrm{Y}$ \\
\hline 19 & J043250.29+294239.5 & J043250.30+294239.7 & M5 & 3.9079747 & 0.0639671 & 7.0587926 & 11.4431 & $\mathrm{Y}$ \\
\hline 19 & J043250.29+294239.5 & J043250.30+294239.7 & L1 & 1.9316559 & 0.0164280 & 3.5155766 & 15.7561 & $\mathrm{Y}$ \\
\hline 20 & J043920.90+254502.1 & J043920.92+254501.9 & M2 & 72.6770935 & 0.4228196 & 85.8005447 & 13.3346 & $\mathrm{Y}$ \\
\hline 20 & J043920.92+254501.8 & J043920.92+254501.9 & M1 & 66.7842712 & 1.5345556 & 112.3731613 & 16.8842 & $\mathrm{Y}$ \\
\hline 21 & J044621.78+172303.1 & J044621.81+172303.5 & M6 & 13.9664001 & 0.0786490 & 15.5491953 & 8.2714 & $\mathrm{Y}$ \\
\hline 21 & J044621.78+172303.1 & J044621.81+172303.5 & M5 & 12.4391394 & 0.0541400 & 13.8067837 & 8.3747 & $\mathrm{Y}$ \\
\hline 22 & J044813.48+292453.5 & J044813.48+292453.5 & M2 & 20.6756344 & 0.6101283 & 82.8008499 & 54.1792 & $\mathrm{Y}$ \\
\hline 22 & J044813.48+292453.4 & J044813.48+292453.5 & M3 & 24.5430584 & 0.8001958 & 85.0495377 & 32.9934 & $\mathrm{Y}$ \\
\hline 23 & J053002.04+121335.8 & J053002.01+121335.7 & G8 & 20.8385754 & 0.1733870 & 42.1097870 & 12.6016 & $\mathrm{Y}$ \\
\hline 23 & J053002.02+121335.6 & J053002.01+121335.7 & K5 & 35.2215080 & 0.0241762 & 126.6769943 & 23.2903 & $\mathrm{Y}$ \\
\hline 24 & J053128.05+120910.2 & J053128.05+120910.2 & G3 & 86.7132111 & 1.5561818 & 63.1558456 & 10.5369 & $\mathrm{Y}$ \\
\hline 24 & J053128.04+120910.3 & J053128.05+120910.2 & F7 & 59.1888199 & 0.0341495 & 338.5122681 & 41.1235 & $\mathrm{Y}$ \\
\hline 25 & J053200.30-045553.8 & J053200.30-045553.8 & B0 & 12.6712179 & 0.0950776 & 13.6715822 & 25.7179 & $\mathrm{Y}$ \\
\hline 25 & J053200.30-045553.8 & J053200.30-045553.8 & B0 & 10.2123613 & 0.1399300 & 11.9934368 & 24.0989 & $\mathrm{Y}$ \\
\hline 26 & J053209.94-024946.7 & J053209.94-024946.7 & K4 & 4.1657314 & 0.0019114 & 62.8156776 & 53.8309 & $\mathrm{Y}$ \\
\hline 26 & J053209.94-024946.7 & J053209.94-024946.7 & K4 & 7.3370047 & 0.0029331 & 52.7106781 & 54.0553 & $\mathrm{Y}$ \\
\hline 27 & J053415.74-063604.6 & J053415.74-063604.5 & K3 & 2.4419281 & 0.0243778 & 12.2068777 & 62.9769 & $\mathrm{Y}$ \\
\hline 27 & J053415.74-063604.6 & J053415.74-063604.5 & K3 & 2.5272474 & 0.0149375 & 12.7578421 & 33.6247 & $\mathrm{Y}$ \\
\hline 28 & J060205.78-011428.3 & J060205.78-011428.2 & G9 & 21.6452446 & 0.0564436 & 71.3180771 & 10.0462 & $\mathrm{Y}$ \\
\hline 28 & J060205.78-011428.3 & J060205.78-011428.2 & G9 & 15.6448870 & 0.0380235 & 46.7075233 & 14.9352 & $\mathrm{Y}$ \\
\hline
\end{tabular}

Note: $\mathrm{Y}$ means that the activity of the star is variable, $\mathrm{N}$ means that the activity of the star is not variable

\subsection{Chromospheric Activity}

The physical mechanism of spectral emission from early and late stars are different. The early-type stars (Oe, Ae and Be), which evolve from dense molecular clouds, has its accretion of dust disks, stellar winds and rapid rotation, leading its $\mathrm{H} \alpha$ emission line originating from the outer atmosphere of the star or stellar envelopes (Golden-Marx et al., 2016; Hou et al., 2016; Ahmed \& Sigut, 2017). The early-type stars with the $\mathrm{H} \alpha$ emission line have 25 infrared source in our sample. The late-type stars produces a spectrum of a series of emission lines (Balmer lines, Ca II H\&K and Ca II IRT). These activity magbe 
due to the stellar chromospheric activity and magnetic activity caused by plage and flare (Zhang et al., 2015; Zhang et al., 2016; Vida et al., 2015; Montes \& Crespo-chacón, 2004).

\section{Summary}

(1) LMAOST and WISE cross-certification have obtained 3762 stars from 158114 infrared stars and 2184 stars from 145905 infrared galaxies.

(2) According to the spectra of active stars emits in the $\mathrm{H} \alpha, \mathrm{H} \beta, \mathrm{H} \delta, \mathrm{H} \gamma$, Ca II H\&K, and Ca II IRT band. We have obtained 390 active stars.

(3) We studied the 390 spectrum of stars and found 77 of them are first observed by LAMOST.

(4) With the help of the equivalent width of the $\mathrm{H} \alpha$ line obtained by the hammer program, We verified that 102 spectra of active stars are variable.

Acknowledgments. This research is supported by the Joint Fund of Astronomy of the NSFC and CAS Grant Nos. 11963002 and U1931132. Guoshoujing Telescope (the Large Sky Area Multi-Object Fiber Spectroscopic Telescope LAMOST) is a National Major Scientific Project built by the Chinese Academy of Sciences.

\section{References}

1. Ahmed, A., Sigut, T. A. A., 2017. MNRAS 471, 3398A.

2. Aumann H. H., et al., 1984. ApJ 278, L23.

3. Covey, K. R., Ivezié,̇̇., et al., 2007. AJ 134, 2398.

4. Catalano, S., Lanza, A. F., et al., 1998. ASPC 154,584.

5. Cui, X. Q., Zhao, Z. H., et al.,2012. RAA 12, 9, 1197.

6. Dhital, S., West, A. A., et al., 2012. AJ 143,67.

7. Egan M. P., Shipman R. F., Price S. D., Carey S. J., Clark F. O., Cohen M., 1998, ApJ 494, L199

8. Frasca, A., Molenda-Żakowicz, J., et al., 2016. A\&A 594, 39.

9. Golden-Marx, Jesse B., Oey, M. S and Lamb, J. B., et al., 2016. APJ 819, 55G.

10. Hou, Wen., Luo, A. Li and Hu, Jing-Yao., 2016. RAA 16,138H.

11. Hawley, S. L., Covey, K. R., Knapp, G. R., et al., 2002. AJ 123,3409.

12. Koenig, X., Hillenbrand, L. A.,et al.,2015,AJ,150,100K

13. Koenig, X. P., Leisawitz, D. T., Benford, D. J., et al., 2012. ApJ 744, 130K

14. Lee, Y. S., Beers, T. C., et al., 2008. AJ 136, 2022.

15. Luo, A. L., Zhang, H. T., et al., 2012. RAA, 12, 9, 1243.

16. Montes,D., Crespo-chacón., et al., 2004. LNEA 1, 119.

17. Oudmaijer, R. D., van der Veen, W. E. C. J., Waters, L. B. F. M., et al. 1992, A\&A, 96, 625

18. Perault, M., et al., 1996. A\&A 315, L165

19. Peretto, N., Fuller G. A., 2010. ApJ 723, 555

20. Rhee, J. H., song I R. zuckerman, B, et al. 2007, ApJ 660, 1556

21. Rathborne J. M., Jackson J. M., Simon R., 2006, ApJ 641, 389

22. Vida, K., Korhonen, H., et al., 2015, A\&A, 580, 64.

23. West, A. A., Morgan, D. P., et al., 2011. AJ 141, 97.

24. Wright, Edward, L., Eisenhardt, Peter, R. M., et al., 2010. AJ 140, 1868.

25. Woolf, V. M., West, A. A., 2012, MNRAS, 422, 1489.

26. Yi, Z. P., Luo, A. L., et al., 2014. AJ 147, 32.

27. Zhang, L. Y., Pi, Q. F., et al., 2015. RAA 15, 252.

28. Zhao, G., Zhao, Z. H., et al., 2012. RAA 12, 7, 723.

29. Zhang, L. Y., Pi, Q. F., et al., 2016. NewA 44, 66. 